\title{
Real Berezin Transform and Asymptotic Expansion for Symmetric Spaces of Compact and Non-compact Type
}

\author{
Miroslav Engliš* and Harald Upmeier
}

ABSTRACT. We obtain formulas for the asymptotic expansion of the Berezin transform on symmetric spaces in terms of invariant differential operators associated with the Peter-Weyl decomposition under the maximal compact subgroup. A unified treatment makes it possible to derive the formulas for the complex (hermitian) as well as for the real case, and for all types of symmetric spaces (non-compact, compact and flat).

\section{Introduction}

The Berezin transform is of central importance in the theory of deformation quantization of complex Kähler manifolds, in particular for the special case of symmetric spaces of hermitian type. In this case the eigenvalues of the Berezin transform are explicitly known, both in the noncompact case of hermitian bounded symmetric domains [UU] and for the compact hermitian symmetric spaces [Z2] arising from duality. Besides the spectral analysis, another important problem is the expansion of the Berezin transform into an asymptotic series of differential operators, as the deformation parameter ("inverse Planck constant") tends to infinity. More precisely, the well-known Toeplitz star- (or Moyal) products have asymptotic expansions which are closely related to that of the (inverse) Berezin transform [EU1], [EU2].

For non-compact symmetric domains, the asymptotic expansion of the Berezin transform was obtained by Arazy and Orsted [AO]. In a separate paper [EU1] we generalized this result to the case of real bounded symmetric domains, where again there is a natural "Berezin" transform which is closely related to the well-known Segal-Bargmann transformations. The dual situtation of compact symmetric spaces (complex or real) was not considered in [AO] or [EU1], [EU2].

The purpose of this paper is to give the asymptotic expansion of the Berezin transform for symmetric spaces of compact and non-compact type, both in the classical complex setting of hermitian symmetric spaces and in the real setting for the Segal-Bargmann type Berezin transform. We present a uniform proof for all cases, showing that the compact type behaves quite similar to the dual non-compact situation. As usual the "flat" case, where the semi-simple covariance group degenerates into a semi-direct product, is included in the computations.

In a separate paper [EU2] we apply these results to obtain asymptotic expansions (in the Peter-Weyl context) for star products in the compact and non-compact situation, as well as

${ }^{*}$ Research supported by the Academy of Sciences of the Czech Republic institutional research plan no. AV0Z10190503; and GA ČR grant no. 201/09/0473. 
for the so-called "star restrictions" which are their real counterparts.

Precursors of the results included here appeared in one of the talks by the first author at the IWOTA09 conference in Guanajuato, Mexico, in Septemer 2009. Both authors thank the organizers for the invitation (which the second author was not able to accept in the end).

\section{Berezin transform for hermitian symmetric spaces}

It is well-known that most Riemannian symmetric spaces, including all the classical ones, have a uniform description in terms of Jordan algebras and Jordan triples. We refer to [EU1], [L] for a detailed discussion of the Jordan theoretic background and notation. For any hermitian Jordan triple $Z$ the (spectral) open unit ball $Z^{-}$is a hermitian bounded symmetric domain whose compact dual $Z^{+}$is a Jordan theoretic analogue of the Grassmann manifold, containing $Z$ as a Zariski open subset, i.e.,

$$
Z^{-} \subset Z \subset Z^{+}
$$

All hermitian symmetric spaces (compact or non-compact) arise this way. (The non-hermitian case will be studied in Section 2.) We define real Lie groups

$$
\begin{aligned}
G^{-} & :=\operatorname{Aut}\left(Z^{-}\right)=\left\{\text {biholomorphic automorphisms of } Z^{-}\right\} \\
G^{+} & =\left\{\text {biholomorphic isometries of } Z^{+}\right\}, \\
G^{0} & =Z \times K \quad \text { (semi-direct product) }
\end{aligned}
$$

where $K=\operatorname{Aut}(Z)=\left\{g \in G^{ \pm}: g(0)=0\right\}$ is the Jordan triple automorphism group.

The three types of hermitian manifolds $Z^{\bullet}=Z^{+}, Z, Z^{-}$give rise to reproducing kernel Hilbert spaces $H_{\nu}^{2}\left(Z^{\bullet}\right)$ of holomorphic functions, which play the role of quantization state spaces. Here $\nu$ is a deformation parameter ("inverse Planck constant"). Let $\mathcal{K} \bullet(z, w)$ denote the reproducing kernel. The Berezin transform (related to the so-called Toeplitz-Berezin quantization calculus $[\mathrm{BMS}])$ is a $G^{\bullet}$-invariant densely defined self-adjoint operator

$$
\mathcal{B}^{\bullet}: L^{2}\left(Z^{\bullet}\right) \rightarrow L^{2}\left(Z^{\bullet}\right)
$$

with integral kernel representation

$$
\left(\mathcal{B}^{\bullet} f\right)(z)=\int_{Z^{\bullet}} d \mu^{\bullet}(w) \frac{\mathcal{K}^{\bullet}(z, w) \mathcal{K}^{\bullet}(w, z)}{\mathcal{K} \bullet(z, z) \mathcal{K} \bullet(w, w)} f(w)
$$

with respect to a suitably normalized $G^{\bullet}$-invariant measure $d \mu^{\bullet}$ specified below.

We will now discuss the three types of hermitian symmetric spaces separately. For $Z \bullet=Z$, the flat case, $\nu>0$ is arbitrary and $H_{\nu}^{2}(Z)$ is the Fock space of all entire functions $\psi \in \mathcal{O}(Z)$ satisfying

$$
\|\psi\|_{\nu}^{2}=\int_{Z} d \mu(z) e^{-\nu(z \mid z)}|\psi(z)|^{2}<+\infty .
$$

Here $(z \mid w)$ denotes the $K$-invariant scalar product on $Z$ normalized by $\left(e_{1} \mid e_{1}\right)=1$ for all minimal tripotents $e_{1} \in Z$, and the "invariant" measure is

$$
d \mu(z)=\nu^{d} \frac{d z}{\pi^{d}}
$$


Here $d$ is the (complex) dimension of $Z$ and $d z$ is the Lebesgue measure for the inner product. The reproducing kernel of $H_{\nu}^{2}(Z)$ is

$$
\mathcal{K}(z, w)=e^{\nu(z \mid w)}
$$

Accordingly, we have

$$
(\mathcal{B} f)(z)=\int_{Z} d \mu(w) \frac{e^{\nu(z \mid w)} e^{\nu(w \mid z)}}{e^{\nu(z \mid z)} e^{\nu(w \mid w)}} f(w)=\nu^{d} \int_{Z} \frac{d w}{\pi^{d}} e^{-\nu(z-w \mid z-w)} f(w) .
$$

In particular,

$$
(\mathcal{B} f)(0)=\nu^{d} \int_{Z} \frac{d w}{\pi^{d}} e^{-\nu(w \mid w)} f(w) .
$$

The basic numerical invariants of an irreducible hermitian Jordan triple $Z$ of rank $r$ can be described via the Peirce decomposition

$$
Z=U \times V=X^{\mathbb{C}} \times V
$$

with respect to a maximal tripotent $e \in Z$ of $\operatorname{rank} r$. Here the Peirce 1 -space $U$ is the complexification of a euclidean Jordan algebra $X$ with unit element $e$, and the Peirce $\frac{1}{2}$-space $V$ carries a Jordan algebra representation of $X$. The "characteristic multiplicities" $a$ and $b$ are defined by

$$
\begin{aligned}
\operatorname{dim}_{\mathbb{R}} X & =\operatorname{dim}_{\mathbb{C}} U=r+\frac{a}{2} r(r-1) \\
\operatorname{dim}_{\mathbb{C}} V & =r b .
\end{aligned}
$$

Hence

$$
\operatorname{dim}_{\mathbb{C}} Z=d=r+\frac{a}{2} r(r-1)+r b .
$$

The genus $p$ of $Z$ is defined by

$$
p=\frac{2 \operatorname{dim} U+\operatorname{dim} V}{r}=2+a(r-1)+b .
$$

For $Z^{\bullet}=Z^{-}$, the bounded case, $\nu$ is a real parameter $>p-1$ and $H_{\nu}^{2}\left(Z^{-}\right)$is the (weighted) Bergman space of holomorphic functions $\psi \in \mathcal{O}\left(Z^{-}\right)$satisfying

$$
\|\psi\|_{\nu}^{2}=\int_{Z^{-}} d \mu^{-}(z) \Delta(z, z)^{\nu}|\psi(z)|^{2}<+\infty
$$

for the $G^{-}$-invariant measure

$$
d \mu^{-}(z)=\frac{\Gamma_{\Omega}(\nu)}{\Gamma_{\Omega}\left(\nu-\frac{d}{r}\right)} \frac{d z}{\pi^{d}} \Delta(z, z)^{-p}
$$

Here $\Delta(z, w)$ is the Jordan triple determinant of $Z, d_{X}:=\operatorname{dim}_{\mathbb{R}} X$ and

$$
\Gamma_{\Omega}(\lambda)=(2 \pi)^{\frac{d_{X}-r}{2}} \prod_{j=1}^{r} \Gamma\left(\lambda_{j}-\frac{a}{2}(j-1)\right)
$$


is the Gindikin $\Gamma$-function of the symmetric cone $\Omega$ of $X$ [FK], [G]. The reproducing kernel of $H_{\nu}^{2}\left(Z^{-}\right)$is given by

$$
\mathcal{K}^{-}(z, w)=\Delta(z, w)^{-\nu}
$$

Therefore

$$
\begin{aligned}
& \left(\mathcal{B}^{-} f\right)(z)=\int_{Z^{-}} d \mu^{-}(w) \frac{\Delta(z, z)^{\nu} \Delta(w, w)^{\nu}}{\Delta(z, w)^{\nu} \Delta(w, z)^{\nu}} f(w) \\
& =\frac{\Gamma_{\Omega}(\nu)}{\Gamma_{\Omega}\left(\nu-\frac{d}{r}\right)} \int_{Z^{-}} \frac{d w}{\pi^{d}} \frac{\Delta(z, z)^{\nu} \Delta(w, w)^{\nu-p}}{\Delta(z, w)^{\nu} \Delta(w, z)^{\nu}} f(w) .
\end{aligned}
$$

In particular

$$
\left(\mathcal{B}^{-} f\right)(0)=\frac{\Gamma_{\Omega}(\nu)}{\Gamma_{\Omega}\left(\nu-\frac{d}{r}\right)} \int_{Z^{-}} \frac{d w}{\pi^{d}} \Delta(w, w)^{\nu-p} f(w) .
$$

For $Z^{\bullet}=Z^{+}$, the compact case, $\nu \in \mathbb{N}$ is a non-negative integer and, as observed in [Z2], $H_{\nu}^{2}\left(Z^{+}\right)$can be realized as a Bergman type space of entire functions $\psi \in \mathcal{O}(Z)$ satisfying

$$
\|\psi\|_{\nu}^{2}=\int_{Z} d \mu^{+}(z) \Delta(z,-z)^{-\nu}|\psi(z)|^{2}<+\infty
$$

for the $G^{+}$-invariant measure

$$
d \mu^{+}(z)=\frac{\Gamma_{\Omega}(\nu+p)}{\Gamma_{\Omega}\left(\nu+p-\frac{d}{r}\right)} \frac{d z}{\pi^{d}} \Delta(z,-z)^{-p} .
$$

This space is finite dimensional, as can be seen from the reproducing kernel

$$
\mathcal{K}^{+}(z, w)=\Delta(z,-w)^{\nu}
$$

Therefore

$$
\begin{aligned}
& \left(\mathcal{B}^{+} f\right)(z)=\int_{Z} d \mu^{+}(w) \frac{\Delta(z,-w)^{\nu} \Delta(w,-z)^{\nu}}{\Delta(z,-z)^{\nu} \Delta(w,-w)^{\nu}} f(w) \\
& =\frac{\Gamma_{\Omega}(\nu+p)}{\Gamma_{\Omega}\left(\nu+p-\frac{d}{r}\right)} \int_{Z} \frac{d w}{\pi^{d}} \frac{\Delta(z,-w)^{\nu} \Delta(w,-z)^{\nu}}{\Delta(z,-z)^{\nu} \Delta(w,-w)^{\nu+p}} f(w) .
\end{aligned}
$$

In particular,

$$
\left(\mathcal{B}^{+} f\right)(0)=\frac{\Gamma_{\Omega}(\nu+p)}{\Gamma_{\Omega}\left(\nu+p-\frac{d}{r}\right)} \int_{Z} \frac{d w}{\pi^{d}} \Delta(w,-w)^{-(\nu+p)} f(w) .
$$

This concludes our case-by-case discussion.

By [U], [FK], the polynomial algebra $\mathcal{P}(Z)$ over $Z$ has a Peter-Weyl decomposition

$$
\mathcal{P}(Z)=\sum_{\boldsymbol{m} \in \mathbb{N}_{+}^{r}} \mathcal{P}_{\boldsymbol{m}}(Z)
$$

under the natural $K$-action. Here $\mathbb{N}_{+}^{r}$ denotes the set of all integer partitions

$$
\boldsymbol{m}=\left(m_{1}, \ldots, m_{r}\right)
$$


of length $\leq r$. We have a corresponding expansion

$$
e^{(z \mid w)}=\sum_{\boldsymbol{m}} E^{\boldsymbol{m}}(z, w)
$$

of the Fischer-Fock kernel in terms of the reproducing kernels $E^{\boldsymbol{m}}(z, w)$ of the finite dimensional subspaces $\mathcal{P}_{\boldsymbol{m}}(Z)$. These functions are polynomials on $Z \times \bar{Z}$ and therefore give rise to constant coefficient bi-differential operators $E^{\boldsymbol{m}}(\partial, \partial)$, where $\partial$ denotes the complex Wirtinger derivative. Specifically, for any fixed $u, v \in Z$,

$$
E^{\boldsymbol{m}}(\partial, \partial) e^{(z \mid v)+(u \mid w)}=E^{\boldsymbol{m}}(u, v) e^{(z \mid v)+(u \mid w)} .
$$

Via the diagonal embedding $z \mapsto(z, \bar{z})$ of $Z$ into $Z \times \bar{Z}$, we also have the operators $\partial_{E} \boldsymbol{m}$ acting on $Z$ by

$$
\partial_{E} \boldsymbol{m} e^{(z \mid v)+(u \mid z)}=E^{\boldsymbol{m}}(u, v) e^{(z \mid v)+(u \mid z)} .
$$

Theorem 1.1. In terms of the bi-differential operators $\partial_{E} \boldsymbol{m}$ on $Z$, the Berezin transform has the asymptotic expansion

$$
\left(\mathcal{B}^{\bullet} f\right)(0)=\sum_{\boldsymbol{m}} c_{\boldsymbol{m}}^{\bullet}(\nu)\left(\partial_{E} \boldsymbol{m} f\right)(0)
$$

at 0 , with $\boldsymbol{m}=\left(m_{1}, \ldots, m_{r}\right)$ running over all partitions of length $\leq r$. Here the coefficients are given by

$$
\begin{aligned}
& c_{\boldsymbol{m}}(\nu)=\frac{1}{\nu^{|\boldsymbol{m}|}} \quad \text { (flat case) } \\
& c_{\boldsymbol{m}}^{-}(\nu)=\frac{1}{(\nu)_{\boldsymbol{m}}} \quad \text { (bounded case) } \\
& c_{\boldsymbol{m}}^{+}(\nu)=\left(\nu+p-\frac{d}{r}\right)_{-\boldsymbol{m}^{*}} \quad(\text { compact case })
\end{aligned}
$$

where $\boldsymbol{m}^{*}:=\left(m_{r}, \ldots, m_{1}\right)$.

Proof. For $K$-invariant integrable functions $f$ on $Z$ we have the polar integration formula [AU2, Proposition 3.4]

$$
\int_{Z} \frac{d x}{\pi^{d}} f(z)=\int_{\Omega} \frac{d x}{\Gamma_{\Omega}\left(\frac{d}{r}\right)} \Delta(x)^{\frac{d}{r}-\frac{d X}{r}} f(\sqrt{x}),
$$

where $\Delta$ is the Jordan algebra determinant of $X[\mathrm{FK}]$. For $x \in \Omega$, we have

$$
\frac{E^{\boldsymbol{m}}(\sqrt{x}, \sqrt{x})}{E^{\boldsymbol{m}}(e, e)}=\phi^{\boldsymbol{m}}(x)
$$

where $\phi^{\boldsymbol{m}}$ is the spherical polynomial of type $\boldsymbol{m}$. Let $\Delta^{\alpha}$ be the conical function on $\Omega$, for $\alpha=\left(\alpha_{1}, \ldots, \alpha_{r}\right)$. By [FK, Theorem VII.1.7] we have

$$
\int_{\Omega \cap(e-\Omega)} d x \Delta^{\alpha}(x) \Delta^{\beta}(e-x)=\frac{\Gamma_{\Omega}\left(\alpha+\frac{d_{X}}{r}\right) \Gamma_{\Omega}\left(\beta+\frac{d_{X}}{r}\right)}{\Gamma_{\Omega}\left(\alpha+\beta+2 \frac{d_{X}}{r}\right)},
$$

and [G, Proposition 2.6] or [AU1, Lemma 5.7] imply

$$
\int_{\Omega} d x \Delta^{\alpha}(x) \Delta^{-\beta}(e+x)=\frac{\Gamma_{\Omega}\left(\alpha+\frac{d_{X}}{r}\right) \Gamma_{\Omega}\left(\beta-\alpha^{*}-\frac{d_{X}}{r}\right)}{\Gamma_{\Omega}(\beta)}
$$


with $\alpha^{*}=\left(\alpha_{r}, \ldots, \alpha_{1}\right)$. In the flat case we have

$$
e^{-\nu(\sqrt{x} \mid \sqrt{x})}=e^{-\nu(x \mid e)}=e^{-(x \mid \nu e)}
$$

for $x \in \Omega$. This yields

$$
\begin{aligned}
& \int_{Z} \frac{d w}{\pi^{d}} e^{-\nu(w \mid w)} \frac{E^{\boldsymbol{m}}(w, w)}{E^{\boldsymbol{m}}(e, e)}=\int_{\Omega} \frac{d x}{\Gamma_{\Omega}\left(\frac{d}{r}\right)} \Delta(x)^{\frac{d}{r}-\frac{d_{X}}{r}} e^{-\nu(\sqrt{x} \mid \sqrt{x})} \frac{E^{\boldsymbol{m}}(\sqrt{x}, \sqrt{x})}{E^{\boldsymbol{m}}(e, e)} \\
& =\int_{\Omega} \frac{d x}{\Gamma_{\Omega}\left(\frac{d}{r}\right)} \Delta(x)^{\frac{d}{r}-\frac{d_{X}}{r}} e^{-\nu(x \mid e)} \phi^{\boldsymbol{m}}(x)=\int_{\Omega} \frac{d x}{\Gamma_{\Omega}\left(\frac{d}{r}\right)} \Delta(x)^{\frac{d}{r}-\frac{d X}{r}} e^{-\nu(x \mid e)} \Delta^{\boldsymbol{m}}(x) \\
& =\int_{\Omega} \frac{d x}{\Gamma_{\Omega}\left(\frac{d}{r}\right)} \Delta^{\boldsymbol{m}+\frac{d}{r}-\frac{d_{X}}{r}}(x) e^{-(x \mid \nu e)}=\frac{\Gamma_{\Omega}\left(\boldsymbol{m}+\frac{d}{r}\right)}{\Gamma_{\Omega}\left(\frac{d}{r}\right)} \Delta^{\boldsymbol{m}+\frac{d}{r}}\left(\nu^{-1} e\right)=\left(\frac{d}{r}\right) \boldsymbol{m} \nu^{-|m|-d},
\end{aligned}
$$

since $\Delta^{\boldsymbol{m}+\frac{d}{r}}$ is homogeneous of total degree $|\boldsymbol{m}|+d$. (The third equality in the chain uses the fact that $d x, \Delta(x)$ and $e^{(x \mid e)}$ are all invariant under the subgroup $L \subset \operatorname{Aut}(\Omega)$ stabilizing $e$, while $\phi^{\boldsymbol{m}}$ is the average of $\Delta^{\boldsymbol{m}}$ over L.) Therefore

$$
\frac{\left(\mathcal{B} E^{\boldsymbol{m}}\right)(0)}{E^{\boldsymbol{m}}(e, e)}=\nu^{d} \int_{Z} \frac{d w}{\pi^{d}} e^{-\nu(w \mid w)} \frac{E^{\boldsymbol{m}}(w, w)}{E^{\boldsymbol{m}}(e, e)}=\nu^{-|m|}\left(\frac{d}{r}\right)_{\boldsymbol{m}} .
$$

In the bounded case we have

$$
\Delta(\sqrt{x}, \sqrt{x})=\Delta(e-x)
$$

for $x \in \Omega \cap(e-\Omega)$. This yields

$$
\begin{aligned}
& \int_{Z^{-}} \frac{d w}{\pi^{d}} \Delta(w, w)^{\nu-p} \frac{E^{\boldsymbol{m}}(w, w)}{E^{\boldsymbol{m}}(e, e)} \\
= & \int_{\Omega \cap(e-\Omega)} \frac{d x}{\Gamma_{\Omega}\left(\frac{d}{r}\right)} \Delta(x)^{\frac{d}{r}-\frac{d_{X}}{r}} \Delta(\sqrt{x}, \sqrt{x})^{\nu-p} \frac{E^{\boldsymbol{m}}(\sqrt{x}, \sqrt{x})}{E^{\boldsymbol{m}}(e, e)} \\
= & \int_{\Omega \cap(e-\Omega)} \frac{d x}{\Gamma_{\Omega}\left(\frac{d}{r}\right)} \Delta(x)^{\frac{d}{r}-\frac{d_{X}}{r}} \Delta(e-x)^{\nu-p} \phi^{\boldsymbol{m}}(x) \\
= & \int_{\Omega \cap(e-\Omega)} \frac{d x}{\Gamma_{\Omega}\left(\frac{d}{r}\right)} \Delta(x)^{\frac{d}{r}-\frac{d_{X}}{r}} \Delta(e-x)^{\nu-p} \Delta^{\boldsymbol{m}}(x) \\
= & \int_{\Omega \cap(e-\Omega)} \frac{d x}{\Gamma_{\Omega}\left(\frac{d}{r}\right)} \Delta^{\boldsymbol{m}+\frac{d}{r}-\frac{d_{X}}{r}}(x) \Delta(e-x)^{\nu-p}=\frac{\Gamma_{\Omega}\left(\boldsymbol{m}+\frac{d}{r}\right)}{\Gamma_{\Omega}\left(\frac{d}{r}\right)} \frac{\Gamma_{\Omega}\left(\nu-\frac{d}{r}\right)}{\Gamma_{\Omega}(\nu+\boldsymbol{m})}
\end{aligned}
$$

since $\nu-p+\frac{d_{X}}{r}=\nu-\frac{d}{r}$. Therefore

$$
\begin{aligned}
& \frac{\left(\mathcal{B}^{-} E^{\boldsymbol{m}}\right)(0)}{E^{\boldsymbol{m}}(e, e)}=\frac{\Gamma_{\Omega}(\nu)}{\Gamma_{\Omega}\left(\nu-\frac{d}{r}\right)} \int_{Z^{-}} \frac{d w}{\pi^{d}} \Delta(w, w)^{\nu-p} \frac{E^{\boldsymbol{m}}(w, w)}{E^{\boldsymbol{m}}(e, e)} \\
& =\frac{\Gamma_{\Omega}(\nu)}{\Gamma_{\Omega}\left(\nu-\frac{d}{r}\right)} \frac{\Gamma_{\Omega}\left(\boldsymbol{m}+\frac{d}{r}\right)}{\Gamma_{\Omega}\left(\frac{d}{r}\right)} \frac{\Gamma_{\Omega}\left(\nu-\frac{d}{r}\right)}{\Gamma_{\Omega}(\nu+\boldsymbol{m})}=\frac{(d / r)_{\boldsymbol{m}}}{(\nu)_{\boldsymbol{m}}} .
\end{aligned}
$$


In the compact case, we have

$$
\Delta(\sqrt{x},-\sqrt{x})=\Delta(e+x)
$$

for $x \in \Omega$. This yields

$$
\begin{aligned}
& \int_{Z} \frac{d w}{\pi^{d}} \Delta(w,-w)^{-(\nu+p)} \frac{E^{\boldsymbol{m}}(w, w)}{E^{\boldsymbol{m}}(e, e)} \\
& =\int_{\Omega} \frac{d x}{\Gamma_{\Omega}\left(\frac{d}{r}\right)} \Delta(x)^{\frac{d}{r}-\frac{d_{X}}{r}} \Delta(\sqrt{x},-\sqrt{x})^{-(\nu+p)} \frac{E^{\boldsymbol{m}}(\sqrt{x}, \sqrt{x})}{E^{\boldsymbol{m}}(e, e)} \\
& =\int_{\Omega} \frac{d x}{\Gamma_{\Omega}\left(\frac{d}{r}\right)} \Delta(x)^{\frac{d}{r}-\frac{d_{X}}{r}} \Delta(e+x)^{-(\nu+p)} \phi^{\boldsymbol{m}}(x) \\
& =\int_{\Omega} \frac{d x}{\Gamma_{\Omega}\left(\frac{d}{r}\right)} \Delta(x)^{\frac{d}{r}-\frac{d_{X}}{r}} \Delta(e+x)^{-(\nu+p)} \Delta^{\boldsymbol{m}}(x) \\
& =\int_{\Omega} \frac{d x}{\Gamma_{\Omega}\left(\frac{d}{r}\right)} \Delta^{\boldsymbol{m}+\frac{d}{r}-\frac{d_{X}}{r}}(x) \Delta(e+x)^{-(\nu+p)} \\
& =\frac{\Gamma_{\Omega}\left(\boldsymbol{m}+\frac{d}{r}\right) \Gamma_{\Omega}\left(\nu+p-\boldsymbol{m}^{*}-\frac{d}{r}\right)}{\Gamma_{\Omega}\left(\frac{d}{r}\right) \Gamma_{\Omega}(\nu+p)} .
\end{aligned}
$$

Therefore

$$
\begin{aligned}
& \frac{\left(\mathcal{B}^{+} E^{\boldsymbol{m}}\right)(0)}{E^{\boldsymbol{m}}(e, e)}=\frac{\Gamma_{\Omega}(\nu+p)}{\Gamma_{\Omega}\left(\nu+p-\frac{d}{r}\right)} \int_{Z} \frac{d w}{\pi^{d}} \Delta(w,-w)^{-(\nu+p)} \frac{E^{\boldsymbol{m}}(w, w)}{E^{\boldsymbol{m}}(e, e)} \\
& =\frac{\Gamma_{\Omega}(\nu+p)}{\Gamma_{\Omega}\left(\nu+p-\frac{d}{r}\right)} \frac{\Gamma_{\Omega}\left(\boldsymbol{m}+\frac{d}{r}\right) \Gamma_{\Omega}\left(\nu+p-\boldsymbol{m}^{*}-\frac{d}{r}\right)}{\Gamma_{\Omega}\left(\frac{d}{r}\right) \Gamma_{\Omega}(\nu+p)}=(d / r)_{\boldsymbol{m}}\left(\nu+p-\frac{d}{r}\right)_{-\boldsymbol{m}^{*}} .
\end{aligned}
$$

In all three cases, since $\mathcal{B}^{\bullet}$ is a $G^{\bullet}$-invariant operator on $Z^{\bullet}$, the localized operator at 0 has a unique expansion

$$
\left(\mathcal{B}^{\bullet} f\right)(0)=\sum_{\boldsymbol{m}} c_{\boldsymbol{m}}^{\bullet}(\nu)\left(\partial_{E} \boldsymbol{m} f\right)(0)
$$

for all functions $f$ which are smooth near $0 \in Z$. This implies for the diagonal $E^{\boldsymbol{m}}(z, z)$

$$
\left(\mathcal{B}^{\bullet} E^{\boldsymbol{m}}\right)(0)=\sum_{\boldsymbol{n}} c_{\boldsymbol{n}}^{\bullet}(\nu) \partial_{E} \boldsymbol{n} E^{\boldsymbol{m}}(0)=c_{\boldsymbol{m}}^{\bullet}(\nu)\left\|E^{\boldsymbol{m}}\right\|_{Z \times \bar{Z}}^{2}=c_{\boldsymbol{m}}^{\bullet}(\nu) E^{\boldsymbol{m}}(e, e)\left(\frac{d}{r}\right)_{\boldsymbol{m}}
$$

It follows that

$$
c_{\boldsymbol{m}}^{\bullet}(\nu)=\frac{1}{(d / r)_{\boldsymbol{m}}} \frac{\mathcal{B}^{\bullet} E^{\boldsymbol{m}}(0)}{E^{\boldsymbol{m}}(e, e)}
$$

has the value specified above.

Remark 1.2. The bounded case of the last theorem recovers the result of $[\mathrm{AO}]$.

\section{Berezin transform for real symmetric spaces}

In order to introduce the "real" counterparts of hermitian symmetric spaces, let $Z_{\mathbb{C}}$ be a hermitian Jordan triple endowed with a triple involution $z \mapsto \tilde{z}$. Then the real form

$$
Z_{\mathbb{R}}=\left\{z \in Z_{\mathbb{C}}: \tilde{z}=z\right\}
$$


is a euclidean Jordan triple which we assume to be irreducible. The associated unit ball $Z_{\mathbb{R}}^{-} \subset Z_{\mathbb{R}}$ and compact dual $Z_{\mathbb{R}}^{+} \supset Z_{\mathbb{R}}$ have the hermitifications $Z_{\mathbb{C}}^{-}$and $Z_{\mathbb{C}}^{+}$, respectively. In summary,

$$
\begin{array}{ccccccc}
Z_{\mathbb{C}}^{-} & \subset & Z_{\mathbb{C}} & \subset & Z_{\mathbb{C}}^{+} \\
\cup & & \cup & & \cup \\
Z_{\mathbb{R}}^{-} & \subset & Z_{\mathbb{R}} & \subset & Z_{\mathbb{R}}^{+} .
\end{array}
$$

Remark 2.1. As a special case of this situation, we obtain the "product" case

$$
\begin{aligned}
& Z_{\mathbb{R}}:=Z_{\text {diag }}=\{(z, \bar{z}): z \in Z\} \subset Z_{\mathbb{C}}:=Z \times \bar{Z}, \\
& Z_{\mathbb{R}}^{-}:=Z_{\text {diag }}^{-}=\left\{(z, \bar{z}): z \in Z^{-}\right\} \subset Z_{\mathbb{C}}^{-}:=Z^{-} \times \bar{Z}^{-}, \\
& Z_{\mathbb{R}}^{+}:=Z_{\text {diag }}^{+}=\left\{(z, \bar{z}): z \in Z^{+}\right\} \subset Z_{\mathbb{C}}^{+}:=Z^{+} \times \bar{Z}^{+},
\end{aligned}
$$

associated with a hermitian Jordan triple $Z$ and the "flip" involution $(z, \bar{w})^{\sim}:=(w, \bar{z})$. In this case (3) takes the form

$$
\begin{array}{cccccc}
Z^{-} \times \bar{Z}^{-} & \subset & Z \times \bar{Z} & \subset & Z^{+} \times \bar{Z}^{+} \\
\cup & & \cup & & \cup \\
Z_{\text {diag }}^{-} & \subset & Z_{\text {diag }} & \subset & Z_{\text {diag }}^{+} .
\end{array}
$$

This is the only case where the complexified spaces are not irreducible $[\mathrm{L}]$. Since this situation is covered by Section 1 , we will assume from now on that $Z_{\mathbb{C}}$ is irreducible.

There exists a maximal tripotent $e \in Z_{\mathbb{R}}\left(\right.$ of rank $\left.r_{\mathbb{R}}\right)$ which is also maximal in $Z_{\mathbb{C}}$ (i.e., of rank $\left.r_{\mathbb{C}}\right)$. Let

$$
Z_{\mathbb{C}}=U_{\mathbb{C}} \times V_{\mathbb{C}}=X_{\mathbb{C}}^{\mathbb{C}} \times V_{\mathbb{C}}
$$

and

$$
Z_{\mathbb{R}}=U_{\mathbb{R}} \times V_{\mathbb{R}}=X_{\mathbb{R}} \times Y_{\mathbb{R}} \times V_{\mathbb{R}}
$$

denote the corresponding Peirce decompositions. Then we have complexifications

$$
U_{\mathbb{C}}=U_{\mathbb{R}}^{\mathbb{C}}, \quad V_{\mathbb{C}}=V_{\mathbb{R}}^{\mathbb{C}}
$$

and the euclidean Jordan algebras $X_{\mathbb{C}}\left(\right.$ of rank $r_{\mathbb{C}}$ ) and $X_{\mathbb{R}}\left(\right.$ of rank $r_{\mathbb{R}}$ ) are related by

$$
\begin{aligned}
X_{\mathbb{R}} & =\left\{x \in X_{\mathbb{C}}: \tilde{x}=x\right\}, \\
i Y_{\mathbb{R}} & =\left\{y \in X_{\mathbb{C}}: \tilde{y}=-y\right\} .
\end{aligned}
$$

Equivalently,

$$
\begin{aligned}
X_{\mathbb{R}} & =\left\{x \in U_{\mathbb{R}}: x^{*}=x\right\}, \\
Y_{\mathbb{R}} & =\left\{y \in U_{\mathbb{R}}: y^{*}=-y\right\}
\end{aligned}
$$

for the Jordan involution ${ }^{*}$ in $U_{\mathbb{C}}$. Writing $e$ as an orthogonal sum of minimal tripotents in $Z_{\mathbb{R}}$ and $Z_{\mathbb{C}}$, respectively, it follows that the normalized inner products $(x \mid y)_{\mathbb{R}}$ in $Z_{\mathbb{R}}$ and $(z \mid w)_{\mathbb{C}}$ in $Z_{\mathbb{C}}$ satisfy $(e \mid e)_{\mathbb{R}}=r_{\mathbb{R}}$ and $(e \mid e)_{\mathbb{C}}=r_{\mathbb{C}}$. Therefore we have the reciprocity

$$
r_{\mathbb{R}}(x \mid y)_{\mathbb{C}}=r_{\mathbb{C}}(x \mid y)_{\mathbb{R}}
$$


for all $x, y \in Z_{\mathbb{R}} \subset Z_{\mathbb{C}}$. Analogous to (1), we have characteristic multiplicities $a_{\mathbb{C}}, b_{\mathbb{C}}$ satisfying

$$
\begin{aligned}
& \operatorname{dim}_{\mathbb{R}} V_{\mathbb{R}}=\operatorname{dim}_{\mathbb{C}} V_{\mathbb{C}}=r_{\mathbb{C}} b_{\mathbb{C}} \\
& \operatorname{dim}_{\mathbb{R}} U_{\mathbb{R}}=\operatorname{dim}_{\mathbb{C}} U_{\mathbb{C}}=r_{\mathbb{C}}+\frac{a_{\mathbb{C}}}{2} r_{\mathbb{C}}\left(r_{\mathbb{C}}-1\right)
\end{aligned}
$$

and hence

$$
\operatorname{dim}_{\mathbb{R}} Z_{\mathbb{R}}=\operatorname{dim}_{\mathbb{C}} Z_{\mathbb{C}}=r_{\mathbb{C}}+\frac{a_{\mathbb{C}}}{2} r_{\mathbb{C}}\left(r_{\mathbb{C}}-1\right)+r_{\mathbb{C}} b_{\mathbb{C}}
$$

Also, the "complex" genus is

$$
p_{\mathbb{C}}=\frac{2 \operatorname{dim}_{\mathbb{C}} U_{\mathbb{C}}+\operatorname{dim}_{\mathbb{C}} V_{\mathbb{C}}}{r_{\mathbb{C}}}=2+a_{\mathbb{C}}\left(r_{\mathbb{C}}-1\right)+b_{\mathbb{C}}
$$

In the real case, we define $a_{\mathbb{R}}, b_{\mathbb{R}}$ via

$$
\begin{aligned}
& \operatorname{dim}_{\mathbb{R}} X_{\mathbb{R}}=r_{\mathbb{R}}+\frac{a_{\mathbb{R}}}{2} r_{\mathbb{R}}\left(r_{\mathbb{R}}-1\right) \\
& \operatorname{dim}_{\mathbb{R}} V_{\mathbb{R}}=r_{\mathbb{R}} b_{\mathbb{R}}
\end{aligned}
$$

and introduce another numerical invariant $c_{\mathbb{R}}$ via

$$
\operatorname{dim}_{\mathbb{R}} Y_{\mathbb{R}}=r_{\mathbb{R}} c_{\mathbb{R}}+\frac{a_{\mathbb{R}}}{2} r_{\mathbb{R}}\left(r_{\mathbb{R}}-1\right)
$$

One can show that this covers all cases of the classification [L], [Z1], [EU1] with one exception (type $D_{2}$ ) which will not be considered here. The "real" genus $p_{\mathbb{R}}$ is defined by

$$
p_{\mathbb{R}}=\frac{p_{\mathbb{C}} r_{\mathbb{C}}}{2 r_{\mathbb{R}}}=\frac{\operatorname{dim}_{\mathbb{R}} U_{\mathbb{R}}}{r_{\mathbb{R}}}+\frac{\operatorname{dim}_{\mathbb{R}} V_{\mathbb{R}}}{2 r_{\mathbb{R}}}=1+c_{\mathbb{R}}+a_{\mathbb{R}}\left(r_{\mathbb{R}}-1\right)+\frac{b_{\mathbb{R}}}{2} .
$$

In terms of $d_{Y}=\operatorname{dim}_{\mathbb{R}} Y_{\mathbb{R}}$ and $d_{X}=\operatorname{dim}_{\mathbb{R}} X_{\mathbb{R}}=\operatorname{dim}_{\mathbb{R}} X_{\mathbb{C}}-d_{Y}$, we have the relations

$$
p_{\mathbb{R}}-\frac{d}{2 r_{\mathbb{R}}}=\frac{d_{X}+d_{Y}}{2 r_{\mathbb{R}}}
$$

and hence

$$
\frac{d}{2 r_{\mathbb{R}}}+\frac{d_{X}}{r_{\mathbb{R}}}-p_{\mathbb{R}}=\frac{d_{X}-d_{Y}}{2 r_{\mathbb{R}}}
$$

Since $Z_{\mathbb{C}}$ is irreducible, we may consider the quantization Hilbert spaces $H_{\nu_{\mathbb{C}}}^{2}\left(Z_{\mathbb{C}}^{\bullet}\right)$ introduced in Section 1, for the appropriate range of parameters $\nu_{\mathbb{C}}$, with reproducing kernel denoted by $\mathcal{K}_{\mathbb{C}}^{\bullet}(z, w)$ for $z, w \in Z_{\mathbb{C}}^{\bullet}$. Besides the "complex" Berezin transform $\mathcal{B}_{\mathbb{C}}^{\bullet}$ on $L^{2}\left(Z_{\mathbb{C}}^{\bullet}, d \mu_{\mathbb{C}}^{\bullet}\right)$ defined as above, for the "invariant" measure $d \mu_{\mathbb{C}}^{\bullet}$ normalized as in Section 1 , there also exists a "real" Berezin transform $\mathcal{B}_{\mathbb{R}}^{\bullet}$, which is a densely defined self-adjoint operator on $L^{2}\left(Z_{\mathbb{R}}^{\bullet}, d \mu_{\mathbb{R}}^{\bullet}\right)$, with integral representation

$$
\left(\mathcal{B}_{\mathbb{R}}^{\bullet} f\right)(z)=\int_{Z_{R}^{\bullet}} d \mu_{\mathbb{R}}^{\bullet}(w) \frac{\mathcal{K}_{\mathbb{C}}^{\bullet}(z, w)}{\mathcal{K}_{\mathbb{C}}^{\bullet}(z, z)^{1 / 2} \mathcal{K}_{\mathbb{C}}^{\bullet}(w, w)^{1 / 2}} f(w) .
$$

Here we use the fact that $\mathcal{K}_{\mathbb{C}}^{\bullet}(z, z)>0$ for all $z \in Z_{\mathbb{R}}^{\bullet}$. For motivation and background concerning the real Berezin transform, cf. [AU1], [Z1]. We define the parameter $\nu_{\mathbb{R}}$ by the condition

$$
\nu_{\mathbb{C}} r_{\mathbb{C}}=2 \nu_{\mathbb{R}} r_{\mathbb{R}}
$$


As in Section 1, we will now discuss the three types of real symmetric spaces separately. For $Z_{\mathbb{R}}^{\bullet}=Z_{\mathbb{R}}$, the real flat case, the invariant measure is

$$
d \mu_{\mathbb{R}}(z)=\nu_{\mathbb{R}}^{d / 2} \frac{d z}{\pi^{d / 2}}
$$

Therefore

$$
\begin{aligned}
& \left(\mathcal{B}_{\mathbb{R}} f\right)(z)=\int_{Z_{\mathbb{R}}} d \mu_{\mathbb{R}}(w) \frac{e^{\nu_{\mathbb{C}}(z \mid w)_{\mathbb{C}}}}{e^{\frac{\nu_{\mathbb{C}}}{2}(z \mid z)_{\mathbb{C}}} e^{\frac{\nu_{\mathbb{C}}}{2}(w \mid w)_{\mathbb{C}}}} f(w) \\
& =\nu_{\mathbb{R}}^{d / 2} \int_{Z_{\mathbb{R}}} \frac{d w}{\pi^{d / 2}} \frac{e^{\nu_{\mathbb{C}}(z \mid w)_{\mathbb{C}}}}{e^{\frac{\nu_{\mathbb{C}}}{2}(z \mid z)_{\mathbb{C}}} e^{\frac{\nu_{\mathbb{C}}^{2}}{2}(w \mid w)_{\mathbb{C}}}} f(w) .
\end{aligned}
$$

In particular,

$$
\left(\mathcal{B}_{\mathbb{R}} f\right)(0)=\nu_{\mathbb{R}}^{d / 2} \int_{Z_{\mathbb{R}}} \frac{d w}{\pi^{d / 2}} e^{-\frac{\nu_{\mathbb{C}}}{2}(w \mid w)_{\mathbb{C}}} f(w) .
$$

For $Z_{\mathbb{R}}^{\bullet}=Z_{\mathbb{R}}^{-}$, the real bounded case, the invariant measure is

$$
d \mu_{\mathbb{R}}^{-}(z)=\frac{\Gamma_{\Omega}\left(\nu_{\mathbb{R}}+\frac{d_{X}-d_{Y}}{2 r}\right)}{\Gamma_{\Omega}\left(\nu_{\mathbb{R}}-p_{\mathbb{R}}+\frac{d_{X}}{r}\right)} \frac{d z}{\pi^{d / 2}} \Delta_{\mathbb{C}}(z, z)^{-p_{\mathbb{C}} / 2},
$$

where $\Delta_{\mathbb{C}}$ denotes the Jordan triple determinant of $Z_{\mathbb{C}}$. Therefore we obtain

$$
\begin{aligned}
& \left(\mathcal{B}_{\mathbb{R}}^{-} f\right)(z)=\int_{Z_{\mathbb{R}}^{-}} d \mu_{\mathbb{R}}^{-}(w) \frac{\Delta_{\mathbb{C}}(z, z)^{\nu_{\mathbb{C}} / 2} \Delta_{\mathbb{C}}(w, w)^{\nu_{\mathbb{C}} / 2}}{\Delta_{\mathbb{C}}(z, w)^{\nu_{\mathbb{C}}}} f(w) \\
& =\frac{\Gamma_{\Omega}\left(\nu_{\mathbb{R}}+\frac{d_{X}-d_{Y}}{2 r}\right)}{\Gamma_{\Omega}\left(\nu_{\mathbb{R}}-p_{\mathbb{R}}+\frac{d_{X}}{r}\right)} \int_{Z_{\mathbb{R}}^{-}} \frac{d w}{\pi^{d / 2}} \frac{\Delta_{\mathbb{C}}(z, z)^{\nu_{\mathbb{C}} / 2} \Delta_{\mathbb{C}}(w, w)^{\left(\nu_{\mathbb{C}}-p_{\mathbb{C}}\right) / 2}}{\Delta_{\mathbb{C}}(z, w)^{\nu_{\mathbb{C}}}} f(w) .
\end{aligned}
$$

In particular

$$
\left(\mathcal{B}_{\mathbb{R}}^{-} f\right)(0)=\frac{\Gamma_{\Omega}\left(\nu_{\mathbb{R}}+\frac{d_{X}-d_{Y}}{2 r}\right)}{\Gamma_{\Omega}\left(\nu_{\mathbb{R}}-p_{\mathbb{R}}+\frac{d_{X}}{r}\right)} \int_{Z_{\mathbb{R}}^{-}} \frac{d w}{\pi^{d / 2}} \Delta_{\mathbb{C}}(w, w)^{\left(\nu_{\mathbb{C}}-p_{\mathbb{C}}\right) / 2} f(w) .
$$

For $Z_{\mathbb{R}}^{\bullet}=Z_{\mathbb{R}}^{+}$, the real compact case, the invariant measure on $Z_{\mathbb{R}}$ is

$$
d \mu_{\mathbb{R}}^{+}(z)=\frac{\Gamma_{\Omega}\left(\nu_{\mathbb{R}}+p_{\mathbb{R}}\right)}{\Gamma_{\Omega}\left(\nu_{\mathbb{R}}+p_{\mathbb{R}}-\frac{d}{2 r}\right)} \frac{d z}{\pi^{d / 2}} \Delta_{\mathbb{C}}(z,-z)^{-p_{\mathbb{C}} / 2}
$$

Therefore

$$
\begin{aligned}
& \left(\mathcal{B}_{\mathbb{R}}^{+} f\right)(z)=\int_{Z_{\mathbb{R}}} d \mu_{\mathbb{R}}^{+}(w) \frac{\Delta_{\mathbb{C}}(z,-w)^{\nu_{\mathbb{C}}}}{\Delta_{\mathbb{C}}(z,-z)^{\nu_{\mathbb{C}} / 2} \Delta_{\mathbb{C}}(w,-w)^{\left(\nu_{\mathbb{C}}+p_{\mathbb{C}}\right) / 2}} f(w) \\
& =\frac{\Gamma_{\Omega}\left(\nu_{\mathbb{R}}+p_{\mathbb{R}}\right)}{\Gamma_{\Omega}\left(\nu_{\mathbb{R}}+p_{\mathbb{R}}-\frac{d}{2 r}\right)} \int_{Z_{\mathbb{R}}} \frac{d w}{\pi^{d / 2}} \frac{\Delta_{\mathbb{C}}(z,-w)^{\nu_{\mathbb{C}}}}{\Delta_{\mathbb{C}}(z,-z)^{\nu_{\mathbb{C}} / 2} \Delta_{\mathbb{C}}(w,-w)^{\left(\nu_{\mathbb{C}}+p_{\mathbb{C}}\right) / 2}} f(w) .
\end{aligned}
$$


In particular

$$
\left(\mathcal{B}_{\mathbb{R}}^{+} f\right)(0)=\frac{\Gamma_{\Omega}\left(\nu_{\mathbb{R}}+p_{\mathbb{R}}\right)}{\Gamma_{\Omega}\left(\nu_{\mathbb{R}}+p_{\mathbb{R}}-\frac{d}{2 r}\right)} \int_{Z_{\mathbb{R}}} \frac{d w}{\pi^{d / 2}} \Delta_{\mathbb{C}}(w,-w)^{-\left(\nu_{\mathbb{C}}+p_{\mathbb{C}}\right) / 2} f(w) .
$$

This concludes our case-by-case discussion.

As in Section 1 , the ( $\mathbb{C}$-valued) polynomial algebra $\mathcal{P}\left(X_{\mathbb{R}}\right) \equiv \mathcal{P}\left(X_{\mathbb{R}}^{\mathbb{C}}\right)$ has a Peter-Weyl decomposition

$$
\mathcal{P}\left(X_{\mathbb{R}}^{\mathbb{C}}\right)=\sum_{\boldsymbol{m} \in \mathbb{N}_{+}^{r_{\mathbb{R}}}} \mathcal{P}_{\boldsymbol{m}}\left(X_{\mathbb{R}}^{\mathbb{C}}\right)
$$

under the natural action of $\operatorname{Aut}\left(X_{\mathbb{R}}^{\mathbb{C}}\right)$, and we have a corresponding kernel expansion

$$
e^{(x \mid y)_{\mathbb{R}}}=\sum_{\boldsymbol{m} \in \mathbb{N}_{+}^{r_{\mathbb{R}}}} E_{\mathbb{R}}^{\boldsymbol{m}}(x, y)
$$

for the (irreducible) euclidean Jordan algebra $X_{\mathbb{R}}$ of rank $r_{\mathbb{R}}$, with $\boldsymbol{m}$ running over all partitions of length $r_{\mathbb{R}}$.

On the other hand, the polynomial algebra $\mathcal{P}\left(Z_{\mathbb{C}}\right)$ of the (irreducible) hermitian Jordan triple $Z_{\mathbb{C}}$ of rank $r_{\mathbb{C}}$ also admits a Peter-Weyl decomposition

$$
\mathcal{P}\left(Z_{\mathbb{C}}\right)=\sum_{\boldsymbol{n} \in \mathbb{N}_{+}^{r_{\mathbb{C}}}} \mathcal{P}_{\boldsymbol{n}}\left(Z_{\mathbb{C}}\right)
$$

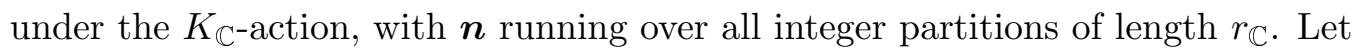

$$
e^{(z \mid w)_{\mathbb{C}}}=\sum_{\boldsymbol{n} \in \mathbb{N}_{+}^{r_{\mathbb{C}}}} E_{\mathbb{C}}^{\boldsymbol{n}}(z, w)
$$

denote the corresponding kernel expansion.

A partition $\boldsymbol{n} \in \mathbb{N}_{+}^{r_{\mathbb{C}}}$ is called even if $\mathcal{P}_{\boldsymbol{n}}\left(Z_{\mathbb{C}}\right)$ contains a non-zero $K_{\mathbb{R}}$-invariant polynomial (which is uniquely determined up to a constant multiple). The results in [Z3] (for tube type domains) and [Z4] (for non-tube type domains) show that with one exception (type A), which we will exclude from consideration here, the even signatures are obtained by "doubling" a signature $\boldsymbol{m} \in \mathbb{N}_{+}^{r_{\mathbb{R}}}$ of length $r_{\mathbb{R}}$. Then the associated $K_{\mathbb{R}}$-invariant polynomial $E^{\boldsymbol{m}} \in \mathcal{P}_{\boldsymbol{m}_{\mathbb{C}}}\left(Z_{\mathbb{C}}\right)$ is uniquely characterized by the condition

$$
E^{\boldsymbol{m}}(x)=E_{\mathbb{R}}^{\boldsymbol{m}}(x, x)=E_{\mathbb{R}}^{\boldsymbol{m}}(e, e) \phi_{\mathbb{R}}^{\boldsymbol{m}}\left(x^{2}\right)
$$

for all $x \in X_{\mathbb{R}} \subset X_{\mathbb{R}}^{\mathbb{C}} \subset Z_{\mathbb{C}}$, where $\phi_{\mathbb{R}}^{\boldsymbol{m}}$ is the spherical polynomial (normalized Jack polynomial) of type $\boldsymbol{m}[\mathrm{FK}]$.

Proposition 2.2. $E^{\boldsymbol{m}}$ has the (complex) Fock space norm

$$
\left\|E^{\boldsymbol{m}}\right\|_{\mathbb{C}}^{2}=E_{\mathbb{R}}^{\boldsymbol{m}}(e, e)\left(\frac{d}{2 r_{\mathbb{R}}}\right) \boldsymbol{m} .
$$


Proof. Put $d_{\boldsymbol{m}}:=\operatorname{dim} \mathcal{P}_{\boldsymbol{m}}\left(X_{\mathbb{R}}^{\mathbb{C}}\right)$. The Shilov boundary $S \subset X_{\mathbb{R}}^{\mathbb{C}}$ is the orbit of $e$ under the group $\operatorname{Aut}\left(X_{\mathbb{R}}^{\mathbb{C}}\right)$. Applying Schur orthogonality and putting $E_{v}^{\boldsymbol{m}}(u):=E_{\mathbb{R}}^{\boldsymbol{m}}(u, v)$ for $u, v \in S$, we obtain for the Fock space inner product $(p \mid q)_{\mathbb{R}}=\left(\partial_{p} q\right)(0)$ on $X_{\mathbb{R}}^{\mathbb{C}}$

$$
\begin{aligned}
& \frac{E_{\mathbb{R}}^{\boldsymbol{m}}(e, e)^{2}}{d_{\boldsymbol{m}}}=\frac{\left(E_{e}^{\boldsymbol{m}} \mid E_{e}^{\boldsymbol{m}}\right)_{\mathbb{R}}^{2}}{d_{\boldsymbol{m}}}=\int_{S} d u\left(E_{e}^{\boldsymbol{m}} \mid E_{u}^{\boldsymbol{m}}\right)_{\mathbb{R}}\left(E_{u}^{\boldsymbol{m}} \mid E_{e}^{\boldsymbol{m}}\right)_{\mathbb{R}} \\
& =\int_{S} d u\left|E_{e}^{\boldsymbol{m}}(u)\right|^{2}=\frac{\left(E_{e}^{\boldsymbol{m}} \mid E_{e}^{\boldsymbol{m}}\right)_{\mathbb{R}}}{\left(d_{X} / r_{\mathbb{R}}\right)_{\boldsymbol{m}}}=\frac{E_{\mathbb{R}}^{\boldsymbol{m}}(e, e)}{\left(d_{X} / r_{\mathbb{R}}\right)_{\boldsymbol{m}}} .
\end{aligned}
$$

This shows

$$
E_{\mathbb{R}}^{\boldsymbol{m}}(e, e)=\frac{d_{\boldsymbol{m}}}{\left(d_{X} / r_{\mathbb{R}}\right) \boldsymbol{m}} .
$$

By [Z4, Lemma 3.3 and Proposition 3.6] we have for the Fock space inner product on $Z_{\mathbb{C}}$

$$
\left\|\frac{E^{\boldsymbol{m}}}{E_{\mathbb{R}}^{\boldsymbol{m}}(e, e)}\right\|_{\mathbb{C}}^{2}=\frac{\left(d_{X} / r_{\mathbb{R}}\right)_{\boldsymbol{m}}\left(d / 2 r_{\mathbb{R}}\right)_{\boldsymbol{m}}}{d_{\boldsymbol{m}}}
$$

Therefore

$$
\left\|E^{\boldsymbol{m}}\right\|_{\mathbb{C}}^{2}=E_{\mathbb{R}}^{\boldsymbol{m}}(e, e)^{2} \frac{\left(d_{X} / r_{\mathbb{R}}\right)_{\boldsymbol{m}}\left(d / 2 r_{\mathbb{R}}\right) \boldsymbol{m}}{d_{\boldsymbol{m}}}=E_{\mathbb{R}}^{\boldsymbol{m}}(e, e)\left(\frac{d}{2 r_{\mathbb{R}}}\right)_{\boldsymbol{m}}
$$

Theorem 2.3. Consider the (holomorphic) differential operators $\partial_{E} \boldsymbol{m}$ on $Z_{\mathbb{R}} \subset Z_{\mathbb{C}}$ induced by the $K_{\mathbb{R}}$-invariant polynomials $E^{\boldsymbol{m}} \in \mathcal{P}_{\boldsymbol{m}_{\mathbb{C}}}\left(Z_{\mathbb{C}}\right)$. Then the real Berezin transform has the asymptotic expansion

$$
\left(\mathcal{B}_{\mathbb{R}}^{\bullet} f\right)(0)=\sum_{\boldsymbol{m}} c_{\boldsymbol{m}}^{\bullet}\left(\nu_{\mathbb{R}}\right)\left(\partial_{E} \boldsymbol{m} f\right)(0),
$$

near 0 , with $\boldsymbol{m}=\left(m_{1}, \ldots, m_{r_{\mathbb{R}}}\right)$ running over all partitions of length $\leq r_{\mathbb{R}}$. Here the coefficients are given by

$$
\begin{aligned}
c_{\boldsymbol{m}}=\nu_{\mathbb{R}}^{-|\boldsymbol{m}|} & \text { (flat case) } \\
c_{\boldsymbol{m}}^{-}=\frac{1}{\left(\nu_{\mathbb{R}}-p_{\mathbb{R}}+\frac{d}{2 r_{\mathbb{R}}}+\frac{d_{X}}{r_{\mathbb{R}}}\right) \boldsymbol{m}} & \text { (bounded case) } \\
c_{\boldsymbol{m}}^{+}=\left(\nu_{\mathbb{R}}+p_{\mathbb{R}}-\frac{d}{2 r_{\mathbb{R}}}\right)_{-\boldsymbol{m}^{*}} & \text { (compact case) }
\end{aligned}
$$

with $\boldsymbol{m}^{*}:=\left(m_{r_{\mathbb{R}}}, \ldots, m_{1}\right)$.

Proof. By [AU2, Proposition 3.4], one has the polar integration formula

$$
\int_{Z_{\mathbb{R}}} \frac{d z}{\pi^{d / 2}} f(z)=\int_{\Omega} \frac{d x}{\Gamma_{\Omega}\left(\frac{d}{2 r_{\mathbb{R}}}\right)} \Delta(x)^{\frac{d}{2 r_{\mathbb{R}}}-\frac{d X}{r_{\mathbb{R}}}} f(\sqrt{x})
$$

for $K_{\mathbb{R}}$-invariant integrable functions $f$ on $Z_{\mathbb{R}}, \Omega$ being the cone of $X_{\mathbb{R}}$ (the proof given there, valid for functions supported in $Z_{\mathbb{R}}^{-}$, extends to the general case by a homogeneity and density argument). For $x \in \Omega$, we have

$$
E^{\boldsymbol{m}}(\sqrt{x})=E_{\mathbb{R}}^{\boldsymbol{m}}(\sqrt{x}, \sqrt{x})=E_{\mathbb{R}}^{\boldsymbol{m}}(e, e) \phi_{\mathbb{R}}^{\boldsymbol{m}}(x) .
$$


In the flat case, we have

$$
e^{-\frac{\nu_{\mathbb{C}}}{2}(\sqrt{x} \mid \sqrt{x})_{\mathbb{C}}}=e^{-\nu_{\mathbb{R}}(\sqrt{x} \mid \sqrt{x})_{\mathbb{R}}}=e^{-\left(x \mid \nu_{\mathbb{R}} e\right)_{\mathbb{R}}}
$$

for $x \in \Omega$. This yields

$$
\begin{aligned}
& \int_{Z_{\mathbb{R}}} \frac{d w}{\pi^{d / 2}} e^{-\frac{\nu_{\mathbb{C}}}{2}(w \mid w)_{\mathbb{C}}} \frac{E^{\boldsymbol{m}}(w)}{E_{\mathbb{R}}^{\boldsymbol{m}}(e, e)} \\
& =\int_{\Omega} \frac{d x}{\Gamma_{\Omega}\left(\frac{d}{2 r_{\mathbb{R}}}\right)} \Delta(x)^{\frac{d}{2 r_{\mathbb{R}}}-\frac{d}{r_{\mathbb{R}}}} e^{-\frac{\nu_{\mathbb{C}}}{2}(\sqrt{x} \mid \sqrt{x})_{\mathbb{C}}} \frac{E^{\boldsymbol{m}}(\sqrt{x})}{E_{\mathbb{R}}^{\boldsymbol{m}}(e, e)} \\
& =\int_{\Omega} \frac{d x}{\Gamma_{\Omega}\left(\frac{d}{2 r_{\mathbb{R}}}\right)} \Delta(x)^{\frac{d}{2 r_{\mathbb{R}}}-\frac{d X}{r_{\mathbb{R}}}} e^{-\nu_{\mathbb{R}}(x \mid e)_{\mathbb{R}}} \phi_{\mathbb{R}}^{\boldsymbol{m}}(x) \\
& =\int_{\Omega} \frac{d x}{\Gamma_{\Omega}\left(\frac{d}{2 r_{\mathbb{R}}}\right)} \Delta(x)^{\frac{d}{2 r_{\mathbb{R}}}-\frac{d X}{r_{\mathbb{R}}}} e^{-\nu_{\mathbb{R}}(x \mid e)_{\mathbb{R}}} \Delta^{\boldsymbol{m}}(x) \\
& =\int_{\Omega} \frac{d x}{\Gamma_{\Omega}\left(\frac{d}{2 r_{\mathbb{R}}}\right)} \Delta^{\boldsymbol{m}+\frac{d}{2 r_{\mathbb{R}}}-\frac{d X}{r_{\mathbb{R}}}}(x) e^{-\left(x \mid \nu_{\mathbb{R}} e\right)_{\mathbb{R}}} \\
& =\frac{\Gamma_{\Omega}\left(\boldsymbol{m}+\frac{d}{2 r_{\mathbb{R}}}\right)}{\Gamma_{\Omega}\left(\frac{d}{2 r_{\mathbb{R}}}\right)} \Delta^{\boldsymbol{m}+\frac{d}{2 r_{\mathbb{R}}}}\left(\nu_{\mathbb{R}}^{-1} e\right)=\left(\frac{d}{2 r_{\mathbb{R}}}\right)_{\boldsymbol{m}} \nu_{\mathbb{R}}^{-|\boldsymbol{m}|-d / 2}
\end{aligned}
$$

since $\Delta^{\boldsymbol{m}+\frac{d}{2 r_{\mathbb{R}}}}$ is homogeneous of total degree $|\boldsymbol{m}|+\frac{d}{2}$. Therefore

$$
\frac{\left(\mathcal{B}_{\mathbb{R}} E^{\boldsymbol{m}}\right)(0)}{E_{\mathbb{R}}^{\boldsymbol{m}}(e, e)}=\nu_{\mathbb{R}}^{d / 2} \int_{Z_{\mathbb{R}}} \frac{d w}{\pi^{d / 2}} e^{-\frac{\nu_{\mathbb{C}}}{2}(w \mid w)_{\mathbb{C}}} \frac{E^{\boldsymbol{m}}(w)}{E_{\mathbb{R}}^{\boldsymbol{m}}(e, e)}=\left(\frac{d}{2 r_{\mathbb{R}}}\right)_{\boldsymbol{m}} \nu_{\mathbb{R}}^{-|\boldsymbol{m}|} .
$$

In the bounded case, we have

$$
\Delta_{\mathbb{C}}(\sqrt{x}, \sqrt{x})=\Delta(e-x)^{r_{\mathbb{C}} / r_{\mathbb{R}}}
$$

for $x \in \Omega \cap(e-\Omega)$. This yields

$$
\begin{aligned}
& \int_{Z_{\mathbb{R}}^{-}} \frac{d w}{\pi^{d / 2}} \Delta \Delta_{\mathbb{C}}(w, w)^{\left(\nu_{\mathbb{C}}-p_{\mathbb{C}}\right) / 2} \frac{E^{\boldsymbol{m}}(w)}{E_{\mathbb{R}}^{\boldsymbol{m}}(e, e)} \\
= & \int_{\Omega \cap(e-\Omega)} \frac{d x}{\Gamma_{\Omega}\left(\frac{d}{2 r_{\mathbb{R}}}\right)} \Delta(x)^{\frac{d}{2 r_{\mathbb{R}}}-\frac{d_{X}}{r_{\mathbb{R}}}} \Delta_{\mathbb{C}}(\sqrt{x}, \sqrt{x})^{\left(\nu_{\mathbb{C}}-p_{\mathbb{C}}\right) / 2} \frac{E^{\boldsymbol{m}}(\sqrt{x})}{E_{\mathbb{R}}^{\boldsymbol{m}}(e, e)} \\
= & \int_{\Omega \cap(e-\Omega)} \frac{d x}{\Gamma_{\Omega}\left(\frac{d}{2 r_{\mathbb{R}}}\right)} \Delta(x)^{\frac{d}{2 r_{\mathbb{R}}}-\frac{d_{X}}{r_{\mathbb{R}}}} \Delta(e-x)^{\nu_{\mathbb{R}}-p_{\mathbb{R}}} \phi_{\mathbb{R}}^{\boldsymbol{m}}(x) \\
= & \int_{\Omega \cap(e-\Omega)} \frac{d x}{\Gamma_{\Omega}\left(\frac{d}{2 r_{\mathbb{R}}}\right)} \Delta(x)^{\frac{d}{2 r_{\mathbb{R}}}-\frac{d}{r_{\mathbb{R}}}} \Delta(e-x)^{\nu_{\mathbb{R}}-p_{\mathbb{R}}} \Delta^{\boldsymbol{m}}(x) \\
= & \int_{\Omega \cap(e-\Omega)} \frac{d x}{\Gamma_{\Omega}\left(\frac{d}{2 r_{\mathbb{R}}}\right)} \Delta^{\boldsymbol{m}+\frac{d}{2 r_{\mathbb{R}}}-\frac{d x}{r_{\mathbb{R}}}}(x) \Delta(e-x)^{\nu_{\mathbb{R}}-p_{\mathbb{R}}}=\frac{\Gamma\left(\boldsymbol{m}+\frac{d}{2 r_{\mathbb{R}}}\right) \Gamma\left(\nu_{\mathbb{R}}-p_{\mathbb{R}}+\frac{d_{X}}{r_{\mathbb{R}}}\right)}{\Gamma_{\Omega}\left(\frac{d}{2 r_{\mathbb{R}}}\right) \Gamma\left(\nu_{\mathbb{R}}+\frac{d_{X}-d_{Y}}{2 r_{\mathbb{R}}}+\boldsymbol{m}\right)}
\end{aligned}
$$


using the relation (2). Therefore

$$
\begin{aligned}
& \frac{\left(\mathcal{B}_{\mathbb{R}}^{-} E^{\boldsymbol{m}}\right)(0)}{E_{\mathbb{R}}^{\boldsymbol{m}}(e, e)}=\frac{\Gamma_{\Omega}\left(\nu_{\mathbb{R}}+\frac{d_{X}-d_{Y}}{2 r_{\mathbb{R}}}\right)}{\Gamma_{\Omega}\left(\nu_{\mathbb{R}}-p_{\mathbb{R}}+\frac{d_{X}}{r_{\mathbb{R}}}\right)} \int_{Z_{\mathbb{R}}^{-}} \frac{d w}{\pi^{d / 2}} \Delta_{\mathbb{C}}(w, w)^{\left(\nu_{\mathbb{C}}-p_{\mathbb{C}}\right) / 2} \frac{E^{\boldsymbol{m}}(w)}{E_{\mathbb{R}}^{\boldsymbol{m}}(e, e)} \\
& =\frac{\Gamma_{\Omega}\left(\nu_{\mathbb{R}}+\frac{d_{X}-d_{Y}}{2 r_{\mathbb{R}}}\right)}{\Gamma_{\Omega}\left(\nu_{\mathbb{R}}-p_{\mathbb{R}}+\frac{d_{X}}{r_{\mathbb{R}}}\right)} \frac{\Gamma\left(\boldsymbol{m}+\frac{d}{2 r_{\mathbb{R}}}\right) \Gamma\left(\nu_{\mathbb{R}}-p_{\mathbb{R}}+\frac{d_{X}}{r_{\mathbb{R}}}\right)}{\Gamma_{\Omega}\left(\frac{d}{2 r_{\mathbb{R}}}\right) \Gamma\left(\nu_{\mathbb{R}}+\frac{d_{X}-d_{Y}}{2 r_{\mathbb{R}}}+\boldsymbol{m}\right)}=\frac{\left(d / 2 r_{\mathbb{R}}\right)_{\boldsymbol{m}}}{\left(\nu_{\mathbb{R}}+\frac{d_{X}-d_{Y}}{2 r_{\mathbb{R}}}\right)_{\boldsymbol{m}}}
\end{aligned}
$$

In the compact case, we have

$$
\Delta_{\mathbb{C}}(\sqrt{x},-\sqrt{x})=\Delta(e+x)^{r_{\mathbb{C}} / r_{\mathbb{R}}}
$$

for $x \in \Omega$ and obtain

$$
\begin{aligned}
& \int_{Z_{\mathbb{R}}} \frac{d w}{\pi^{d / 2}} \Delta_{\mathbb{C}}(w,-w)^{-\left(\nu_{\mathbb{C}}+p_{\mathbb{C}}\right) / 2} \frac{E^{\boldsymbol{m}}(w)}{E_{\mathbb{R}}^{\boldsymbol{m}}(e, e)} \\
& =\int_{\Omega} \frac{d x}{\Gamma_{\Omega}\left(\frac{d}{2 r_{\mathbb{R}}}\right)} \Delta(x)^{\frac{d}{2 r_{\mathbb{R}}}-\frac{d X}{r_{\mathbb{R}}}} \Delta_{\mathbb{C}}(\sqrt{x},-\sqrt{x})^{-\left(\nu_{\mathbb{C}}+p_{\mathbb{C}}\right) / 2} \frac{E_{\mathbb{R}}^{\boldsymbol{m}}(\sqrt{x})}{E_{\mathbb{R}}^{\boldsymbol{m}}(e, e)} \\
& =\int_{\Omega} \frac{d x}{\Gamma_{\Omega}\left(\frac{d}{2 r_{\mathbb{R}}}\right)} \Delta(x)^{\frac{d}{2 r_{\mathbb{R}}}-\frac{d X}{r_{\mathbb{R}}}} \Delta(e+x)^{-\nu_{\mathbb{R}}-p_{\mathbb{R}}} \phi_{\mathbb{R}}^{\boldsymbol{m}}(x) \\
& =\int_{\Omega} \frac{d x}{\Gamma_{\Omega}\left(\frac{d}{2 r_{\mathbb{R}}}\right)} \Delta(x)^{\frac{d}{2 r_{\mathbb{R}}}-\frac{d X}{r_{\mathbb{R}}}} \Delta(e+x)^{-\nu_{\mathbb{R}}-p_{\mathbb{R}}} \Delta^{\boldsymbol{m}}(x) \\
& =\int_{\Omega} \frac{d x}{\Gamma_{\Omega}\left(\frac{d}{2 r_{\mathbb{R}}}\right)} \Delta^{\boldsymbol{m}+\frac{d}{2 r_{\mathbb{R}}}-\frac{d_{X}}{r_{\mathbb{R}}}}(x) \Delta(e+x)^{-\nu_{\mathbb{R}}-p_{\mathbb{R}}}=\frac{\Gamma_{\Omega}\left(\boldsymbol{m}+\frac{d}{2 r_{\mathbb{R}}}\right) \Gamma_{\Omega}\left(\nu_{\mathbb{R}}+p_{\mathbb{R}}-\boldsymbol{m}^{*}-\frac{d}{2 r_{\mathbb{R}}}\right) .}{\Gamma_{\Omega}\left(\frac{d}{2 r_{\mathbb{R}}}\right) \Gamma_{\Omega}\left(\nu_{\mathbb{R}}+p_{\mathbb{R}}\right)} .
\end{aligned}
$$

Therefore

$$
\begin{aligned}
& \frac{\left(\mathcal{B}_{\mathbb{R}}^{+} E^{\boldsymbol{m}}\right)(0)}{E_{\mathbb{R}}^{\boldsymbol{m}}(e, e)}=\frac{\Gamma_{\Omega}\left(\nu_{\mathbb{R}}+p_{\mathbb{R}}\right)}{\Gamma_{\Omega}\left(\nu_{\mathbb{R}}+p_{\mathbb{R}}-\frac{d}{2 r_{\mathbb{R}}}\right)} \int_{Z_{\mathbb{R}}} \frac{d w}{\pi^{d / 2}} \Delta_{\mathbb{C}}(w,-w)^{-\left(\nu_{\mathbb{C}}+p_{\mathbb{C}}\right) / 2} \frac{E^{\boldsymbol{m}}(w)}{E_{\mathbb{R}}^{\boldsymbol{m}}(e, e)} \\
& =\frac{\Gamma_{\Omega}\left(\nu_{\mathbb{R}}+p_{\mathbb{R}}\right)}{\Gamma_{\Omega}\left(\nu_{\mathbb{R}}+p_{\mathbb{R}}-\frac{d}{2 r_{\mathbb{R}}}\right)} \frac{\Gamma_{\Omega}\left(\boldsymbol{m}+\frac{d}{2 r_{\mathbb{R}}}\right) \Gamma_{\Omega}\left(\nu_{\mathbb{R}}+p_{\mathbb{R}}-\boldsymbol{m}^{*}-\frac{d}{2 r_{\mathbb{R}}}\right)}{\Gamma_{\Omega}\left(\frac{d}{2 r_{\mathbb{R}}}\right) \Gamma_{\Omega}\left(\nu_{\mathbb{R}}+p_{\mathbb{R}}\right)}=\left(\frac{d}{2 r_{\mathbb{R}}}\right)_{\boldsymbol{m}}\left(\nu_{\mathbb{R}}+p_{\mathbb{R}}-\frac{d}{2 r_{\mathbb{R}}}\right)_{-\boldsymbol{m}^{*}} .
\end{aligned}
$$

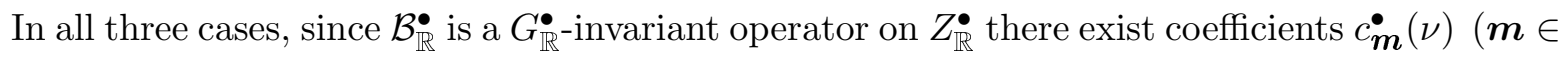
$\mathbb{N}_{+}^{r}$ ) such that

$$
\left(\mathcal{B}_{\mathbb{R}}^{\bullet} f\right)(0)=\sum_{\boldsymbol{m}} c_{\boldsymbol{m}}^{\bullet}\left(\nu_{\mathbb{R}}\right)\left(\partial_{E} \boldsymbol{m} f\right)(0)
$$

for all functions $f$ which are smooth near $0 \in Z_{\mathbb{R}}$. This implies

$$
\left(\mathcal{B}_{\mathbb{R}}^{\bullet} E^{\boldsymbol{m}}\right)(0)=\sum_{\boldsymbol{k}} c_{\boldsymbol{k}}^{\bullet}\left(\partial_{E^{\boldsymbol{k}}} E^{\boldsymbol{m}}\right)(0)=c_{\boldsymbol{m}}^{\bullet}\left\|E^{\boldsymbol{m}}\right\|_{\mathbb{C}}^{2}=c_{\boldsymbol{m}}^{\bullet} E_{\mathbb{R}}^{\boldsymbol{m}}(e, e)\left(\frac{d}{2 r_{\mathbb{R}}}\right)_{\boldsymbol{m}}
$$

It follows that

$$
c_{\boldsymbol{m}}^{\bullet}=\frac{1}{\left(d / 2 r_{\mathbb{R}}\right)_{\boldsymbol{m}}} \frac{\left(\mathcal{B}_{\mathbb{R}}^{\bullet} E^{\boldsymbol{m}}\right)(0)}{E_{\mathbb{R}}^{\boldsymbol{m}}(e, e)}
$$

has the values specified above. 
Remark 2.4. Since

$$
\left(\nu_{\mathbb{R}}-p_{\mathbb{R}}+\frac{d}{2 r_{\mathbb{R}}}+\frac{d_{X}}{r_{\mathbb{R}}}\right)_{\boldsymbol{m}}=\left(\nu_{\mathbb{R}}+\frac{d_{X}-d_{Y}}{2 r_{\mathbb{R}}}\right)_{\boldsymbol{m}},
$$

the bounded case of the last theorem is in complete agreement with Theorem 14 of [EU1] (up to a factor of $\left(2 r_{\mathbb{R}} / r_{\mathbb{C}}\right)^{2|\boldsymbol{m}|}$, which is due to our different normalization of the inner product $(\cdot \mid \cdot)_{\mathbb{C}}$ with respect to $(\cdot \mid \cdot)_{\mathbb{R}}$.)

\section{References}

[AO] J. Arazy, B. Ørsted: Asymptotic expansions of Berezin transforms, Indiana Univ. Math. J. 49 (2000), 7-30.

[AU1] J. Arazy, H. Upmeier: Covariant symbolic calculi on real symmetric domains, Singular integral operators, factorization and applications, pp. 1-27, Oper. Theory Adv. Appl. 142, Birkhuser, Basel, 2003.

[AU2] J. Arazy, H. Upmeier: A one-parameter calculus for symmetric domains, Math. Nachr. 280 (2007), 939-961.

[BMS] M. Bordemann, E. Meinrenken, M. Schlichenmaier: Toeplitz quantization of Kähler manifolds and $g l(N), N \rightarrow \infty$, limits, Comm. Math. Phys. 165 (1994), 281-296.

[EU1] M. Engliš, H. Upmeier: Toeplitz quantization and asymptotic expansions for real bounded symmetric domains, Math. Z. (to appear), http://www.math.cas.cz/englis/70.pdf.

[EU2] M. Engliš, H. Upmeier: Toeplitz quantization and asymptotic expansion: Peter-Weyl decomposition, (submitted), preprint, http://www.math.cas.cz/englis/75.pdf.

[FK] J. Faraut, A. Korányi, Analysis on Symmetric Cones, Clarendon Press, Oxford, 1994.

[G] S. Gindikin: Analysis on homogeneous domains, Uspekhi Mat. Nauk 19 (1964), 3-92; Russian Math. Surveys 19 (1964), 1-89.

[L] O. Loos, Bounded symmetric domains and Jordan pairs, University of California, Irvine, 1977.

[U] H. Upmeier: Toeplitz Operators and Index Theory in Several Complex Variables, Birkhäuser 1996.

[UU] H. Upmeier, A. Unterberger: The Berezin transform and invariant differential operators, Comm. Math. Phys. 164 (1994), 563-597.

[Z1] G. Zhang: Berezin transform on real bounded symmetric domains, Trans. Amer. Math. Soc. 353 (2001), 3769-3787.

[Z2] G. Zhang: Berezin transform on compact Hermitian symmetric spaces, Manuscripta Math. 97 (1998), 371-388. 
[Z3] G. Zhang: Branching coefficients of holomorphic representations and Segal-Bargmann transform, J. Funct. Anal. 195 (2002), 306-349.

[Z4] G. Zhang: Degenerate principal series representations and their holomorphic extensions, preprint (2007), arXiv:math/0711.1480.

Mathematics Institute AS CR, Žitná 25, 11567 Prague 1, Czech Republic and Mathematics Institute, Silesian University in Opava, Na RybníčKu 1, 74601 Opava, Czech Republic

Email address: englis@math.cas.cz

Fachbereich Mathematik, Universität Marburg, D-35032 Marburg, Germany

Email address: upmeier@mathematik.uni-marburg.de 